\title{
Designing a Multi-Functional Vehicle for Aerial Hydraulic Maintenance
}

\author{
Zang Hong-jiao \\ China University of Geosciences (Beijing) \\ Beijing, China \\ Yu Xiang * \\ China University of Geosciences (Beijing) \\ Beijing, China \\ Email: yuxiang@cugb.edu.cn
}

\author{
Zhang Ren-cai \\ China University of Geosciences (Beijing) \\ Beijing, China \\ Xu Shuang-cheng \\ China University of Geosciences (Beijing) \\ Beijing, China
}

\begin{abstract}
Hydraulic maintenance vehicle for aviation is a critical ground device to ensure the flight safety and the provision of hydraulic oil. A novel multi-functional vehicle for aerial hydraulic maintenance is designed for proposing a maintenance approach for modern aircraft. Aiming at issues of existing ground maintenance devices, this work attempts to meet three design requirements as follows: (i) All purpose in one vehicle. This design offers a solution integrating fueling, pressure supplying and cleaning in one vehicle, so as to overcome single function issue of the existing devices. (ii) Active hydraulic oil purification. A high-speed centrifugal separation method has been employed to dealing with low efficiency and easy pollution of existing passive oil purification. (iii) Good motility. Aiming at two issues of large occupation and frequent operation transformation, the hydraulic system, power system, control system and auxiliary mechanism are installed on a drag-and-drop vehicle body to share a compact structure, flexible mobility, and convenient operation.
\end{abstract}

Keywords-aviation hydraulic system; oil contamination; ground maintenance; passive purification; multi-function

\section{INTRODUCTION}

Flight safety is directly correlated with people life and property safety, and frequent flight accidents in recent years have drawn an intensive attention. Mechanical failure is one main factor affecting flight safety ${ }^{[1]}$. Providing energy for control and operation systems of aircraft, the hydraulic system suffers from the most frequent mechanical failures, accounting about $40 \%$ of total mechanical failures ${ }^{[2]}$. Meanwhile, the hydraulic system generally has a complex structure and is located in a closed space. Once an accident occurs, it is very difficult to conduct positioning and maintenance, and thus easily induces disastrous accidents. As a result, ground maintenance is a key approach to reduce the hydraulic failures and to ensure the flight safety ${ }^{[3,4]}$. The ground maintenance for aircraft has three main functions: (i) Fueling of hydraulic oil tank; (ii) Providing pressure for performance testing and debugging of hydraulic control system $^{[5]}$; (iii) Cleaning aerial hydraulic system.
Two main issues of the national ground maintenance devices are: (i) Can only achieve a single function. During filling and pressure supplying operations, maintenance requires multiple devices to operate in sequence. Such maintenance process needs large floor space and frequent switching operations, and is easy to induce mal-operations. (ii) Passive filter purification. The purification and oil contamination level of existing ground maintenance devices relies on the passive oil filter, and the filter elements need to be cleaned or replaced frequently to reduce pollution. Such frequent cleaning and replacement of the filter elements not only increase the costs with evidence, but also cause hydraulic system pollution easily. Meanwhile, the foreign maintenance products subjects to high cost, poor parts supply and technology blockade on China ${ }^{[6-8]}$.

The public has put forward an increasing requirement for maintenance devices to ensure the flight safety and the provision of hydraulic oil ${ }^{[9]}$. Aiming at the existing problems, a novel aerial hydraulic maintenance vehicle is designed to meet three design requirements as expected: (i) All propose in one vehicle. The maintenance vehicle integrates the functions of fueling, pressure supplying and cleaning in one vehicle. (ii) Active hydraulic oil purification. The maintenance vehicle can in-situ separate solid, liquid and gaseous pollutants from hydraulic oil, and does not need to use the filter elements. (iii) Good motility. The drag-and-drop vehicle body is adopted to enjoy a compact structure and convenient operation.

\section{DESIGN REQUIREMENTS AND SOLUTIONS}

The hydraulic circuit is a critical link for the design success. The design ideas are settled down after many repeating trials: (i) All purpose in one vehicle. The hydraulic circuits of fueling, pressure supplying and cleaning are respectively designed, and then all circuits are integrated into a hydraulic circuit for multi-functional maintenance vehicle. (ii) Active hydraulic oil purification. A high-speed centrifugal separation method is adopted to realize active purification of hydraulic oil. 


\section{A. All purpose in one vehicle}

\section{1) Fueling Mode}

In the fueling mode, purified oil is used for fueling hydraulic system of the plane and clean oil tank on ground to ensure the oil sufficiency of plane and maintenance vehicle.

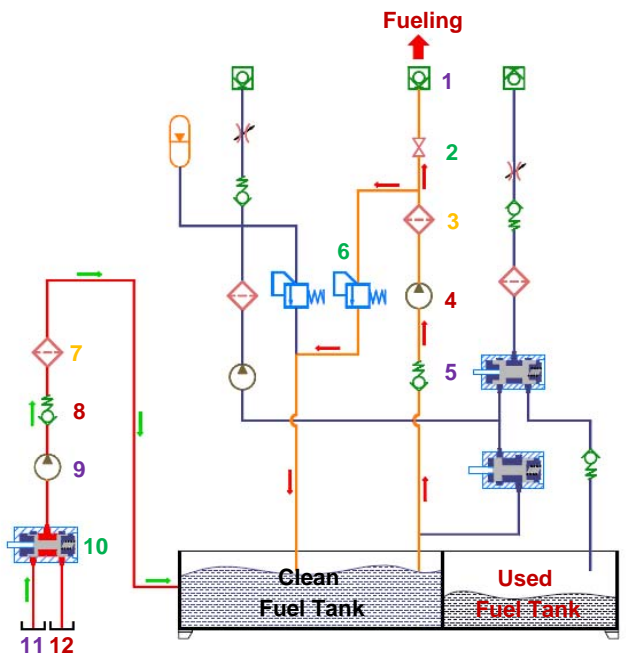

Figure 1. Schematic diagram of fueling circuit 1 Fueling valve connector; 2 Stop valve; 3,7 Oil filter; 4 Oil pump 5,8 Check valve; 6 Relief valve; 9 Hydraulic pump; 10 Reverse valve; 11 Standard oil; 12 Purified oil

Fig. 1 shows hydraulic circuit of maintenance vehicle in fueling mode. The oil flow direction follows order of the red arrow for a clear reason. Switching on stop valve 2, activate pump 4 to pump the purified oil into the fueling pipeline, and the oil goes from check valve 5, oil filter 3 , fueling valve connector 1 to the plane's hydraulic system. Extra oil returns to clean oil tank on the ground through relief valve 6 , in case that the oil tank in the plane has reached its needed quantity.

Refueling pipeline are indicated using green arrows in Fig. 1. Prior to refueling, the refueling connector shall be connected with external standard oil tanks 11 or oil purifier outlet 12 . During refueling, select the oil source through reverse valve 10, and activate hydraulic oil pump 9 to pump standard oil into the clean oil tank through check valve 8 and oil filter 7 , to form a refueling process.

2) Pressure supplying Mode

In pressure supplying mode, provide the plane's hydraulic system with the hydraulic power, by means of replacing the hydraulic source inside the plane with that of the maintenance vehicle.

Fig. 2 shows hydraulic circuit of maintenance vehicle in pressure-supply mode. The oil flow direction follows order of the orange arrow. Before operation, reverse valve 5 lies at its left position, and reverse valve 6 lies at its right cut-off position, making oil-return pipeline connect with pressure-supply pipeline. Rotating speed of main pump 7 shall lower down to avoid over oil pressure in the hydraulic pipeline of the vehicle and the plane. During operation, activating main pump 7 , the oil in the plane goes to main pump 7 through oil-return valve connector 1 , restriction valve 2 , check valve 3 , oil filter 4 and reverse valve 5 in the oil-return pipeline; in the pressure-supply pipeline, the high-pressure oil pumped by main pump passes from filter 8 , reverse valve 10 , restriction valve 11 , and pressure-supply valve connector 12 to hydraulic system of the plane. In case that the pressure in the plane's hydraulic system is too high, switch on relief valve 9, and the extra oil will return to the oil tank through relief valve 9 .

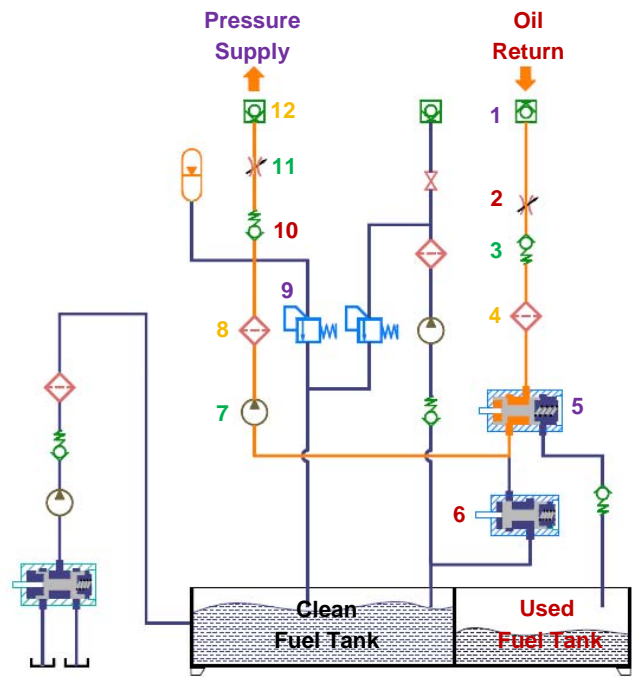

Figure 2. Schematic diagram of pressure-supply circuit 1 Oil-return valve connector; 2 Restriction valve; 3,10 Check valve; 4,8 Oil filter; 5,6 Reverse valve; 7 Main pump; 9 Relief valve;

11 Restriction valve; 12 Pressure-supply valve connector

\section{3) Cleaning Mode}

In cleaning mode, replace polluted oil in hydraulic system of the plane with purified oil circulation of mass flow to develop the cleanliness of the hydraulic system.

Fig. 3 shows hydraulic circuit of maintenance vehicle in cleaning mode. Before operation, reverse valve 10 lies at its right position and reverse valve 11 lies at its left position, making the oil-return and pressure-supply pipeline connect with the ground oil tank. During operation, as indicated by the orange line, activating main pump 1, the oil in the clean oil tank will be pumped to pressure-supply pipeline through reverse valve 11 , and the oil in the pressure-supply pipeline goes from oil filter 2, check valve 3 , restriction valve 4 and fueling valve connector to the plane hydraulic system. In addition, the oil in the plane returns to the used oil tank in sequency of through oil-return valve connector 6 , restriction valve 7 , check valve 8 , oil filter 9 , reverse valve 10 , and check valve 12 . The cleaning process can realize the replacement of oil in the plane's hydraulic system as well as the cleaning of pipeline and accessories. In this case, main pump 1 uses full-flow output, the relief valve in the pressure-supply pipeline is in a normally open state, and the plane's hydraulic system is at the full-flow cleaning state. 


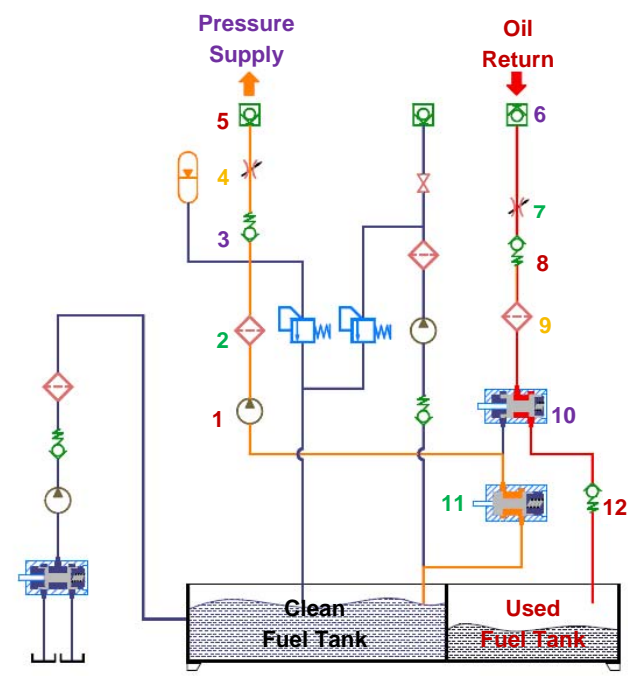

Figure 3. Schematic diagram of cleaning circuit 1 Main pump; 2,9 Oil filter; 3,8,12 Check valve; 4,7 Restriction valve; 5 Pressure-supply valve connector; 6 Oil-return valve connector; 10,11 Reverse valve

\section{4) Integration of Three Functions}

All purpose in a vehicle can be realized like this: (i) Respectively design the hydraulic circuits of fueling, pressure-supply and cleaning; (ii) Integrate all modes circuits and simplify the components to obtain the multifunctional hydraulic circuit of maintenance vehicle.

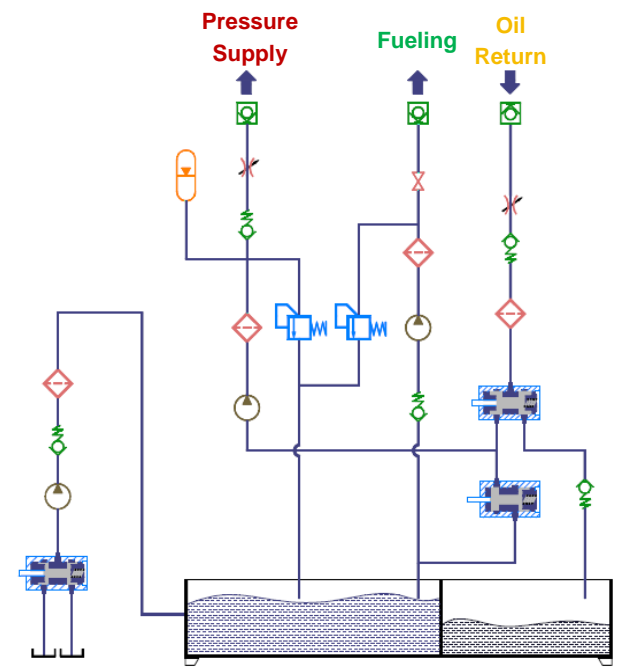

Figure 4. Schematic diagram of multi-functional hydraulic circuit

Fig. 4 shows multi-functional hydraulic circuit of the maintenance vehicle. Before maintenance, as indicated by the blue arrow, connect the connectors of pressure-supply, fueling and oil-return valves of the maintenance vehicle with the plane's oil tank valve together. During maintenance, fueling mode shall be initially activated to fuel the plane's hydraulic system and the ground clean oil tank. When the fuel capacity is sufficient, fueling mode shall be switched to pressure-supply mode to provide hydraulic power for the inspection of the plane's hydraulic system. Subsequently, the mode shall be switched to cleaning, using purified oil of mass flow to purify the plane's hydraulic system.

\section{B. Active hydraulic Oil Purification}

The critical point of designing the oil purification is to choose a suitable method of hydraulic oil purification. At present, common oil purification normally employs passive filter purification to remove oil pollutants, and its problems are: (i) Only solid pollutants in the oil can be passively intercepted and absorbed, whereas the liquid pollutants and gaseous pollutants cannot be removed for a thorough purification; (ii) Filter elements need to be cleaned and replaced frequently, which leads to a higher cost, and the pollutants outside are easier to be blended into the hydraulic system; (iii) The allowable pollution quantity of filter is limited, and the quantity can only be expanded through increasing the element size.

After many trials and comparisons, we decide to use high-speed centrifugal separation method to actively purify the plane's hydraulic system. Having a large capacity and prolonged maintenance period, this patented technique can realize all-around removal of solid, liquid and gaseous pollutants without filter loss ${ }^{[10]}$.

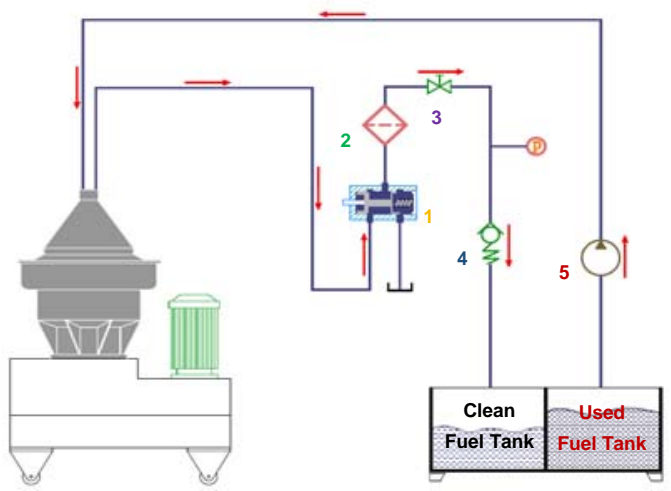

Figure 5. Schematic diagram of working principle for oil purification

1 Reverse valve; 2 Oil filter; 3 Pressure control valve; 4 Check valve; 5 Self-suction booster pump

Fig.5 shows working principle of the active purification. The oil inlet of clean oil tank is connected with that of centrifugal oil purifier through inlet pipes. The oil flow direction follows order of the orange arrow. During operation, activating self-suction booster pump 5, the used oil will be pumped to the centrifugal purifier for separation and purification. In outlet pipeline, the separated clean oil moves from reverse valve 1 , oil filter 2 , and check valve 4 to the clean oil tank. Pressure control valve 3 is installed in outlet pipes to adjust the pressure of incoming oil to the clean oil tank. As the addition of active purification function, the maintenance vehicle does not need to be equipped with special device to purify the plane's hydraulic oil. And the centrifugal oil purifier avoids the pollution brought by repeated output of oil and increases the maintenance efficiency.

\section{STRUCTURAL COMPOSITION}

The overall structure design of the multi-functional maintenance vehicle is fulfilled through function positioning, which is based on above theoretical design including fueling, pressure testing, cleaning and purification of hydraulic system. 


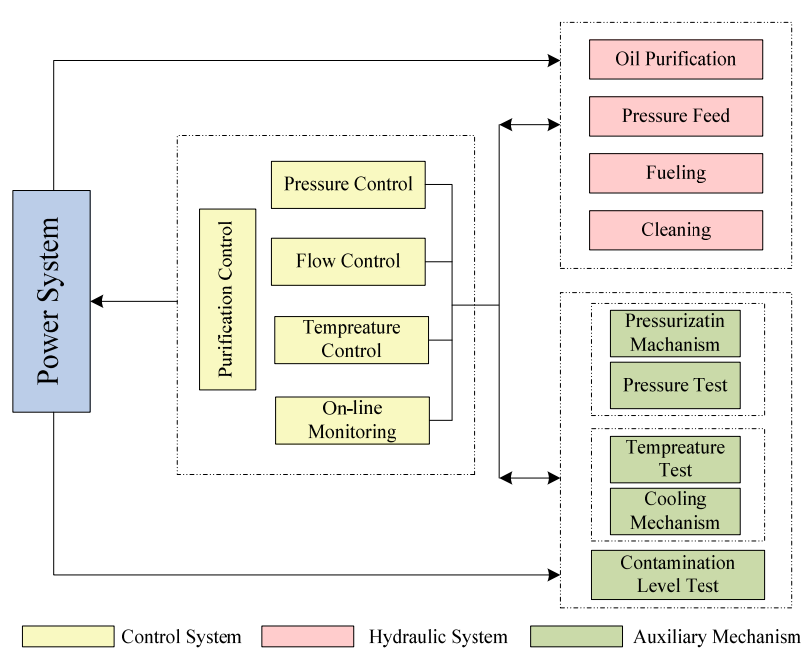

Figure 6. Overall structure diagram of ground maintenance vehicle

Fig. 6 shows overall structure of the ground maintenance vehicle. The overall structure is consisted of hydraulic system, control system, power system and auxiliary mechanism. During operation, the hydraulic systems of the vehicle and the aircraft will form a closed circuit to provide hydraulic pressure source for processes of cleaning, fueling, pressure feed and active purification ${ }^{[11,12]}$. Using the control system can in-situ monitor oil performance parameters of the start, stop, switching in four functional modes to guarantee an exact and reliable maintenance. Using the power system can control the hydraulic pump to provide the high-pressure oil required for maintenance, and to ensure that the oil has enough flow rate and pressure. The auxiliary mechanism is adopted to cooperate with other systems to ensure an effective operation of the maintenance vehicle. In view of simple-structure and economy, the maintenance vehicle employs drag-and-drop type, and the type can select alternative manual drag or vehicle pull on a basis of actual using distance.

\section{CONCLUSIONS}

The hydraulic maintenance vehicle for aviation is a critical ground device to ensure the flight safety and the supply of hydraulic oil. This design offers a solution integrating fueling, pressure supplying and cleaning in one vehicle, so as to overcome single function issue of the existing devices. Meanwhile, an active hydraulic oil purification method is adopted to solve the problems of low efficiency and easy pollution for existing passive oil purification. The overall vehicle structure is designed through theoretical analyses, and is consisted of hydraulic system, power system, control system and auxiliary mechanism and vehicle body.

This design is expected to meet three requirements: (i) All purpose in one vehicle. Under action of the control system, the reverse valves can change the oil direction, and allows switching among three modes of fueling, pressure supply and cleaning. (ii) Active hydraulic oil purification. The high-speed centrifugal separation method is adopted to separate the solid, liquid and gaseous pollutants from hydraulic oil in a real-time, which avoids the filter material loss and pollution caused by repeated oil ingress and egress during the passive purification process. (iii) Good motility. The hydraulic system, power system, control system and auxiliary mechanism are installed on a drag-and-drop vehicle body to share a compact structure, flexible mobility, and convenient operation.

\section{ACKNOWLEDGMENT}

This work was supported by the Fundamental Research Funds for Chinese Central Universities.

\section{REFERENCES}

[1] TAMASI G, DEMICHELA M. An assessment of risk and safety in civil aviation [J]. Reliability Engineering and System Safety, 2011, 96(8): 892-899.

[2] FUKUI A, TAKEDA M. Aircraft actuator hydraulic system: US, 3, 741, 076 [P]. 2013-1-4 [2013-12-19].

[3] ZHANG Wen-guang, LIN Guo-min. Analysis of aircraft hydraulic system failures [J]. Advanced Materials Research, 2014, 989: 2947-2950.

[4] LI Chuang, ZHANG Ming, WEI Xiao-hui, et al. Design, analysis and experimental validation for hydraulic extension retraction system of aircraft landing gears [J]. Journal of Nanjing University of Aeronautics and Astronautics, 2014, 46(2): 225-231.

[5] ZHANG Yan-jun, LI Zheng-wei, YU Zi-wang, et al. Evaluation of developing an enhanced geothermal heating system in northeast China: Field hydraulic stimulation and heat production forecast [J]. Energy and Buildings, 2015, 88: 1-14.

[6] BAILEY R M, HOSTETLER R W, BARNES $\mathrm{K} N$, et al. Experimental validation: Subscale aircraft ground facilities and integrated test capability [C] // Proceedings of AIAA Guidance Navigation, and Control Conference and Exhibit. Portugal: [s. n.], 2005: 110-114.

[7] YE Zhen, WANG Jin-fu. A preventive method of fault for hydraulic system-cleaning methods [J]. China Metal Forming Equipment and Manufacturing Technology, 2011, 4: 49-51.

[8] WANG Shao-ping, ZHOU Ru-sheng, JIAO Zong-xia. Current research and developing trends on fault diagnosis of hydraulic systems [J]. Chinese Journal of Mechanical Engineering, 2006, 42(9): 9-14.

[9] FENG Sheng, CHENG Wei-hong, CHAO Zhi-qiang, et al. Nonintervened and in situ testing method of a certain type of amphibious armored equipment hydraulic system [J]. Journal of Academy of Armored Force Engineering, 2009, 23(4): 40-44.

[10] JAUNCEY P F. Oil/Water separator: US, 3,300,684 [P]. 2011-1121 [2012-5-24].

[11] CHEN Yong, YU Jin-yong, ZHANG Chun-ping, et al. The malfunction analysis and improvement of hydraulic test bench for undercarriage retraction actuator of a helicopter [J]. Advanced Materials Research, 2014, 926: 482-485.

[12] MONTAZERI G, NASIRI M, RAJABI M, JAMSHIDFARD M Actuator based hardware in the loop testing of a jet engine fuel control unit in flight conditions [J]. Simulation Modelling Practice and Theory, 2012, 2(1): 165-77. 